\title{
Modified adiabatic approximation for a hydrogen atom moving in a magnetic field
}

\author{
V.G. BEZCHASTNOV \\ Ioffe Institute of Physics and Technology, 194021, St.Petersburg, Russia \\ A.Y. POTEKHIN \\ Ioffe Institute of Physics and Technology, 194021, St.Petersburg, Russia
}

\begin{abstract}
Motion of a hydrogen atom across the magnetic field shifts center of electron density distribution. For strong magnetic fields, the radiative transitions can be considered in the modified adiabatic approximation in which the shifts are taken into account. The method is illustrated by calculating the photoionization cross sections.
\end{abstract}

\section{1:1 Introduction}

The presence of hydrogen atoms affects radiative transfer in cool atmospheres of neutron stars with strong magnetic fields $B=10^{11}-10^{13} \mathrm{G}$ (Shibanov et $a l$., 1993). If $\gamma \equiv B /\left(2.35 \times 10^{9} \mathrm{G}\right) \gg 1$, the atomic structure and radiative transitions have been considered by many authors for non-moving atoms (see, e.g., Potekhin and Pavlov, 1993 and the references therein). The effects of atomic motion across $\vec{B}$ have been studied using the adiabatic approximation (Gorkov and Dzyaloshinsky, 1968), variational calculations (Vincke et al., 1992), and the perturbation theory (Pavlov and Mészáros, 1993). For strong fields, it is natural to use the basis of the states of free charged particles (Landau functions). In the adiabatic approximation, one keeps the main term of the wave function expansion. In the traditional approach, the transverse part of the wave function is localized in a magnetic well. However, the real shift of the electron density from the Coulomb center cannot coincide with the shift of the magnetic well. We 
consider matrix elements for radiative transitions assuming that the adiabatic wave functions for initial and final states are arbitrary shifted. This approach is applied to calculating the photoionization cross sections.

\subsection{Basic equations}

The structure of the hydrogen atom with generalized momentum $\hbar \vec{K}$ in the magnetic field $\vec{B}=(0,0, B)$ is determined by the Schrödinger equation

$$
\begin{aligned}
& {\left[\frac{\hbar^{2} K_{z}^{2}}{2 M}+(1-\eta)^{2} \frac{\hbar^{2} K_{\perp}^{2}}{2 M}+\frac{\hat{p}_{z}^{2}}{2 \mu}+\hat{H}_{\perp}+\right.} \\
& \left.(1-\eta) \frac{e \hbar}{M c}\left(\vec{K}_{\perp} \times \vec{B}\right) \vec{r}_{\perp}-\frac{e^{2}}{\left|\vec{r}+\eta \vec{r}_{0}\right|}-E\right] \psi_{\vec{K}}^{(\eta)}(\vec{r})=0,
\end{aligned}
$$

where $E$ is the atomic energy, $M=m_{e}+m_{p}, \mu=\left(m_{e} m_{p}\right) / M ; m_{\varepsilon}$ and $m_{p}$ are electron and proton masses, respectively, $\vec{r}=\vec{r}_{e}-\vec{r}_{p}-\eta \vec{r}_{0}$ is the shifted relative co-ordinate, $\vec{r}_{0}=-\left(c \hbar / e B^{2}\right) \vec{K}_{\perp} \times \vec{B}$ and $\hat{p}_{z}=-i \hbar \nabla_{z}$. The Hamiltonian

$$
\hat{H}_{\perp}=\frac{\hat{p}_{\perp}^{2}}{2 \mu}+\frac{e^{2} B^{2}}{8 \mu c^{2}} r_{\perp}^{2}+\frac{e æ}{c \mu} \vec{B}\left(\vec{r}_{\perp} \times \hat{\vec{p}}_{\perp}\right)
$$

$\left(æ=\left(m_{p}-m_{\varepsilon}\right) / 2 M\right.$ and $\left.\hat{\vec{p}}_{\perp}=-i \hbar \nabla_{\perp}\right)$ corresponds to harmonic motion in the " $x-y$ "-plane; $\eta$ is an arbitrary parameter. Each atomic state is described by the family of eigenfunctions with various $\eta$,

$$
\psi_{\vec{K}}^{\left(\eta^{\prime}\right)}(\vec{r})=\exp \left\{i æ\left(\eta-\eta^{\prime}\right) \vec{K}_{\perp} \vec{r}_{\perp}\right\} \psi_{\vec{K}}^{(\eta)}\left(\vec{r}-\vec{r}_{*}\right),
$$

where $\vec{r}_{*}=\left(\eta-\eta^{\prime}\right) \vec{r}_{0}$. If $\gamma \gg 1$, one may construct the adiabatic solution

$$
\psi_{\vec{K}, i}^{(\eta)}(\vec{r})=g_{\vec{K}, i}^{(\eta)}(z) \Phi_{n, N}\left(\vec{r}_{\perp}\right) .
$$

Here $\Phi_{n, N}$ is an eigenfunction of $\hat{H}_{\perp}, n=0,1,2, \ldots$ and $N=0,1,2, \ldots$ enumerate the electron and proton Landau levels, respectively. Then the full set of atomic quantum numbers is $|\vec{K}, i\rangle \equiv|\vec{K}, n, N, \epsilon\rangle$, where $\epsilon$ (positive or negative) determines the "longitudinal" energy. The motion across $\vec{B}$ shifts the electron density distribution in the direction of $\vec{r}_{0}$ - see Vincke $c t$ al. (1992). The accuracy of Eq. (4) should be better, if $\eta$ corresponds to the real shift. Estimates and calculations for the bound states (Ipatova $t$ t al., 1984, Vincke et al., 1992) show that $\eta \simeq 0$ at $K_{\perp}<K_{c}$, and $\eta \simeq 1$ at $K_{\perp}>K_{c}$, the critical value $K_{c}$ being larger for higher binding energy. 


\subsection{Radiative transitions}

Let us assume that the initial and final states of the moving atom are defined by (4) with different shifts, $\eta$ and $\eta^{\prime}$, respectively. Then the dipole matrix element $\vec{D}_{f, i}=\langle\vec{K}, f|\vec{r}| \vec{K}, i\rangle$ contains the factors

$$
I_{\perp}=\left\langle\Phi_{n^{\prime}, N^{\prime}}\left(\vec{r}_{\perp}+\vec{r}_{*}\right)\left|\exp \left\{i æ\left(\eta-\eta^{\prime}\right) \vec{K}_{\perp} \vec{r}_{\perp}\right\}\right| \Phi_{n, N}\left(\vec{r}_{\perp}\right)\right\rangle,
$$

whose direct calculation is complicated because $\vec{r}_{*} \neq 0$. However, using the shift transformation

$$
\Phi_{n^{\prime}, N^{\prime}}\left(\vec{r}_{\perp}+\vec{r}_{*}\right)=\exp \left(\frac{i}{\hbar} \vec{r}_{*} \hat{\vec{p}}_{\perp}\right) \Phi_{n^{\prime}, N^{\prime}}\left(\vec{r}_{\perp}\right)
$$

we may rewrite Eq. (5) in the form

$$
I_{\perp}=\left\langle n^{\prime}, N^{\prime}\left|\exp \left\{\frac{i}{\hbar}\left(\eta^{\prime}-\eta\right)\left(\vec{r}_{0} \hat{\vec{p}}_{\perp}+æ \hbar \vec{K}_{\perp} \hat{\vec{r}}_{\perp}\right)\right\}\right| n, N\right\rangle
$$

where the initial and final states belong to the same Landau basis. For this basis, $\hat{\vec{p}}_{\perp}$ and $\dot{\vec{r}}_{\perp}$ can be expressed through the annihilation and creation operators, which have the following properties:

$$
\begin{array}{ll}
\hat{a}|n, N\rangle=\sqrt{n}|n-1, N\rangle, & \hat{a}^{+}|n, N\rangle=\sqrt{n+1}|n+1, N\rangle, \\
\hat{A}|n, N\rangle=\sqrt{N}|n, N-1\rangle, & \hat{A}^{+}|n, N\rangle=\sqrt{N+1}|n, N+1\rangle, \\
{\left[\hat{a}, \hat{a}^{+}\right]=\left[\hat{A}, \hat{A}^{+}\right]=1 .}
\end{array}
$$

Expanding the exponent in Eq. (7) over powers of the operators $\hat{a}, \hat{A}, \hat{a}^{+}$ and $\hat{A}^{+}$, we obtain

$$
I_{\perp}=\mathrm{e}^{-i\left(n^{\prime}-N^{\prime}-n+N\right) \phi} F_{n^{\prime}, n}\left(\left(m_{e} / M\right)^{2} u\right) F_{N^{\prime}, N}\left(\left(m_{p} / M\right)^{2} u\right),
$$

where

$$
\phi=\pi / 2+\arctan \left(K_{y} / K_{x}\right), \quad u=\left(\eta-\eta^{\prime}\right)^{2}\left(a_{M} K_{\perp}\right)^{2} / 2,
$$

$a_{M}=\sqrt{c \hbar / e B}$ and $F_{k^{\prime}, k}$ is a normalized Laguerre function. This yields the cyclic components of $\vec{D}_{f, i}$ :

$$
\begin{aligned}
& D_{+1} \equiv\left(D_{x}+i D_{y}\right) / \sqrt{2}=-a_{M^{2}} \mathrm{e}^{-i\left(n^{\prime}-N^{\prime}-n-1+N\right) \phi} \times \\
& {\left[\sqrt{n+1} F_{n^{\prime}, n+1} F_{N^{\prime}, N}-\sqrt{N} F_{n^{\prime}, n} F_{N^{\prime}, N-1}\right]\left\langle g_{\vec{K}, f}^{\left(\eta^{\prime}\right)}(z) \mid g_{\vec{K}, i}^{(\eta)}(z)\right\rangle,} \\
& D_{-1} \equiv\left(D_{x}-i D_{y}\right) / \sqrt{2}=-a_{M^{2}} \mathrm{e}^{-i\left(n^{\prime}-N^{\prime}-n+1+N\right) \phi} \times \\
& {\left[\sqrt{n} F_{n^{\prime}, n-1} F_{N^{\prime}, N}-\sqrt{N+1} F_{n^{\prime}, n} F_{N^{\prime}, N+1}\right]\left\langle g_{\vec{K}, f}^{\left(\eta^{\prime}\right)}(z) \mid g_{\vec{K}, i}^{(\eta)}(z)\right\rangle,}
\end{aligned}
$$


Bezchastnov \& Potekhin: A hydrogen atom moving in a magnetic field

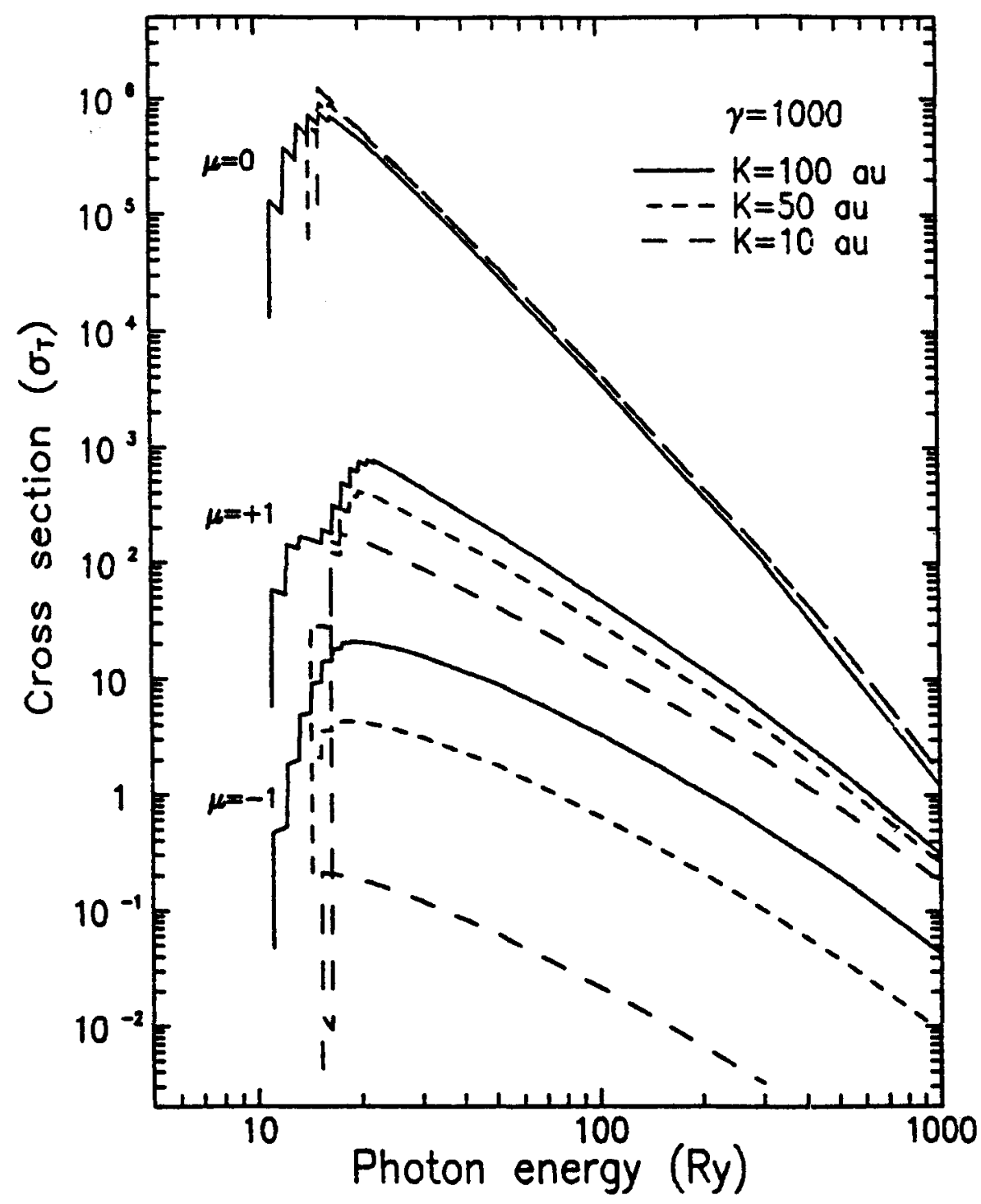

Fig. 31.1 Comparison of traditional $\left(\eta^{\prime}=\eta=1\right.$, long-dashed lines) and modified $\left(\eta^{\prime}=1, \eta=0\right.$, short-dashed lines) adiabatic photoionization cross sections of moving hydrogen atom with more accurate results (solid lines) for $B=2.35 \times 10^{12} \mathrm{G}$ and $K_{\perp}=50$ a.u. $\mu=0$ and $\mu=+1$ correspond to longitudinal and right circular polarizations of photons, respectively.

$$
D_{0} \equiv D_{z}=\mathrm{e}^{-i\left(n^{\prime}-N^{\prime}-n+N\right) \phi} F_{n^{\prime}, n} F_{N^{\prime}, N}\left\langle g_{\vec{K}, f}^{\left(\eta^{\prime}\right)}(z)|z| g_{\vec{K}, i}^{(\eta)}(z)\right\rangle
$$

(the arguments of the $F$-functions with low-case and capital indices are the same as in Eq. (9)).

Photoionization of the moving hydrogen atom can be determined by the transitions between the states with different shifts. If $K_{\perp}<K_{c}$, then the ground-state electron is localized near the nucleus (according to Vincke $\bullet$ 
al. 1992, $K_{c} \sim 150$ a.u. for $\left.\gamma=1000\right)$. Hence the adiabatic approximation with $\eta=0$ is appropriate for this initial state. For the final (continuum) states, the representation with $\eta^{\prime}=1$ should be applied in order to make the Hamiltonian (1) axially symmetric at large $z$. Eqs. (11) allow one to reduce the dipole matrix elements to the "longitudinal" overlap integrals to be found numerically. This gives good agreement with more advanced results computed with the exact wave functions of the initial state (Fig. 1). For comparison, we have calculated the conventional adiabatic cross sections $\left(\eta^{\prime}=\eta=1\right)$. As seen in Fig. 1, this approach underestimates strongly the transition rates at $K_{\perp}<K_{c}$.

\subsection{Discussion}

Motion of the hydrogen atom across the magnetic field makes the problem essentially three-dimensional, which complicates the numerical approach. The adiabatic approximation simplifies the solution reducing the problem to one-dimensional. We have shown that the accuracy of such consideration is determined by a proper choice of shifts $\left(\eta\right.$ and $\left.\eta^{\prime}\right)$. The dependence $\eta\left(B, K_{\perp}, i\right)$ is not yet known. However, we know that $\eta \simeq 0$ at $K_{\perp}<K_{c}$, and $\eta \simeq 1$ at $K_{\perp}>K_{c}$, where $K_{c}$ is a function of the magnetic field and atomic state. An analytic estimate of the critical value $K_{c}$ is thought to be useful.

We are grateful to G.G. Pavlov and D.G. Yakovlev for useful discussions. The work was partly supported by the ESO C\&EE Grant A-01-068.

\section{References}

Gorkov L.P. and Dzyaloshinsky I.E., Sov. Phys.-JETP 26, 449, (1968)

Ipatova I.P., Maslov A.Y. and Subashiev A.V., Sov. Phys.-JETP 60, 1037, (1984)

Pavlov G.G. and Mészáros P., Astrophys. J., accepted, (1993)

Potekhin A.Y. and Pavlov G.G., Astrophys. J. 407, 330, (1993)

Shibanov Y.A. et al, Physics of Isolated Pulsurs. Proc. Los Alamos Workshop, eds. K. Van Riper et al. Cambridge University Press, pp. 174-81, (1993)

Vincke M., Le Dourneuf M. and Baye D., J. Phys. B. 25, 2787, (1992) 